\title{
Condiciones laborales de docentes de educación primaria
}

\author{
Ricardo Fuentes Reza \\ Salvador Ruiz López \\ Carmen Griselda Loya Ortega
}

Centro de Investigación y Docencia Centro Chihuahuense de Estudios de Posgrado

\section{Resumen}

e presenta información cuantitativa sobre las percepciones que tie$\checkmark$ nen los docentes de educación primaria de escuelas federalizadas y estatales de los municipios de Ahumada, Chihuahua y Juárez, Estado de Chihuahua, México, sobre su situación laboral en el contexto de la RIEB.

En esta parte del estudio se aprecia una tendencia sobre el aumento de trabajo de los docentes en sus diferentes funciones derivado de la implementación de la RIEB además de que sus condiciones laborales no se modificaron; también se aprecia una tendencia positiva sobre su satisfacción laboral y sobre su propia valoración de la función que desempeñan.

Palabras clave: Percepción, profesores, educación primaria, condiciones de trabajo, reforma curricular

\section{Introducción}

Las condiciones laborales son los términos con los cuales se conviene realizar un trabajo determinado entre el empleador y el empleado. Esto se da a partir de un trabajo que amerita la contratación de personas, regularmente en un espacio físico concreto y que se mide en unidades de tiempo determinado o por unidades de producción. Este convenio puntualiza una relación específica entre quien contrata la fuerza de trabajo y el que la vende, que es el trabajador o empleado quien porta potencialmente algo que pondrá en acción en esa unidad de tiempo determinada realizando una o varias tareas indispensables para lograr la meta laboral.

Esas múltiples regulaciones que establece el empleador y acepta el empleado/a determinan las condiciones laborales de éste/a. Es decir, por medio de las regula- 
ciones del trabajo, se delimita, contiene y condiciona lo que se precisa hacer; esto da lugar a la definición de los derechos y obligaciones de las partes que componen esta relación.

Dichas regulaciones se cruzan con la existencia humana del trabajador y se gestan respuestas específicas y diversas de parte de él o ella, en el contexto en que se desempeña, de lo cual se deriva una multiplicidad de matices a la hora de concretar la encomienda laboral.

Para el caso de los docentes de escuelas públicas de educación primaria, federalizadas y estatales, también tienen determinadas condiciones laborales y cumplen las características arriba señaladas. Ese marco normativo laboral lo constituye la Ley Federal de Trabajadores al Servicio del Estado, Reglamentaria del Apartado B) del Artículo 123, de la Constitución de los Estados Unidos Mexicanos (2014) donde se consignan los derechos y obligaciones de estos trabajadores.

El trabajo docente en sus diferentes funciones está regulado además por reglamentos y normas específicas, entre ellos el Reglamento de las Condiciones Generales de Trabajo del Personal de la Secretaría de Educación Pública (1946) además de los reglamentos para el funcionamiento de las escuelas, los diversos Acuerdos publicados en el Diario Oficial de la Federación y demás disposiciones, lo cual determina un perfil de condiciones laborales explícitas, pero hay otras que están implícitas que se urden discretamente de tal forma que a veces también resulta difícil establecer los límites de unas y otras.
En el contexto de la RIEB que corresponde a una reforma curricular, se integran nuevas regulaciones como las que se derivan de los estándares curriculares, de desempeño docente y de gestión que determinan algunas condiciones laborales ya existentes y generan otras nuevas. Así encontramos que "las reformas educativas de los últimos años, [...], han traído nuevas exigencias profesionales para los docentes, sin la necesaria adecuación de las condiciones de trabajo" (Oliveira, Goncalves, Melo, 2004, p. 188).

De hecho, la RIEB deja de lado en términos generales el asunto de las condiciones laborales de los docentes en sus diferentes funciones dando por supuesto que ellos asumirán en forma automática las nuevas exigencias curriculares, aun cuando modifiquen algunas de sus condiciones laborales preexistentes.

De esta forma,

Esos cambios también han implicado un aumento del tiempo dedicado por el profesor para atender a estudiantes y padres, además de las reuniones con colegas para la planificación y evaluación del trabajo. Tales cambios repercuten directamente sobre la organización del trabajo escolar, pues exhiben más tiempo de labor del maestro, mismo que, si no es asumido objetivamente en su jornada, acaba traduciéndose en la intensificación laboral, que le obliga a responder a un número mayor de exigencias en menos tiempo (Oliveira, Goncalves, Melo, 2004:189).

Es precisamente hasta diciembre de 2012 en que se realiza una profunda reforma laboral y administrativa que entre otras 
cosas incluye aspectos que no contempló la reforma curricular denominada RIEB que se implementa desde 2004 en preescolar, 2006 en secundaria y en 2009 en educación primaria. Sin embargo, la presente investigación se realiza antes de la reforma laboral y administrativa de diciembre de 2012 y revisa entonces este ángulo donde se realiza esta reforma curricular donde quedó invisible el tema de las condiciones laborales de los docentes del nivel básico (Fuentes, 2010).

Por lo cual no se consideran las nuevas circunstancias que se derivan de la reforma laboral y administrativa de diciembre de 2012, que sin duda representa una nueva reformulación de las condiciones laborales de los trabajadores de la educación que trastoca situaciones y prácticas que se venían realizando por lo menos desde cinco décadas atrás en nuestro país.

El presente trabajo forma parte de una investigación más amplia denominada "La educación primaria; dinámica institucional y reforma educativa" que se lleva a cabo en las escuelas primarias federalizadas y estatales de los municipios de Ahumada, Chihuahua y Juárez.

El objeto de estudio fue abordado desde la interrogante ¿cuáles son las percepciones que tienen los docentes de educación primaria en sus diferentes funciones educativas con relación a su situación laboral en el contexto de la RIEB?

Conocer lo que está pasando con las y los profesores en el nivel de educación primaria que atienden poco más del $60 \%$ del servicio educativo del estado de Chihuahua con relación a su situación laboral en el contexto de la Reforma Integral de la Educación Básica (RIEB), fue el propósito de este esfuerzo investigativo. A partir de identificar rasgos del perfil demográfico de los docentes, directores, asesores técnico pedagógicos, supervisores y jefes de sector, además de describir algunas características de sus funciones, así como puntualizar ciertos elementos de su carrera docente en el nivel y analizar las percepciones que ellos tienen sobre su condición laboral.

\section{Método}

Se trata de una investigación con un alcance descriptivo de enfoque cuantitativo en el que se utilizó el método de la encuesta.

Para ello se diseñaron, pilotearon y aplicaron cuestionarios docentes, directores, ATP, supervisión y también a alumnos de quinto y sexto grado de educación primaria. Los cuestionarios se pilotearon bajo el test-retest con una diferencia de 15 días y se sometieron al proceso de confiabilidad con la aplicación de la "r" de Pearson para ítems de opción múltiple y Alpha de Cronbach para las escalas que se utilizaron; así, se obtuvieron índices de correlación de 0.81 en "r" de Pearson y 0.78 para Alpha de Cronbach.

La aplicación de los cuestionarios fue simultánea y en condiciones similares en un lapso de dos semanas. El procesamiento y análisis estadístico de los datos se llevó a cabo con el apoyo del programa computarizado sPSs. De forma simultánea a todo este proceso, se llevó a cabo una parte del trabajo etnográfico en ocho escuelas primarias federalizadas estatales y posterior- 
mente en 45 nuevas escuelas para penetrar con mayor profundidad la realidad en estudio y avanzar en la fase cualitativa de la investigación, cuyo análisis iniciará después de concluir la fase cuantitativa del estudio.

Se consultaron 1,112 docentes frente a grupo, a la vez 169 directores/as de escuelas primarias, 106 auxiliares técnico pedagógicos (ATP) y 69 supervisores escolares de un universo de 9,747 docentes con alguno de estos roles, los cuales laboran en las escuelas primarias públicas -federales y estatales- de los municipios de Juárez, Chihuahua y Ahumada; es decir, en esta investigación se consultaron en total 1,456 profesores y profesoras que realizan alguna de estas funciones y 1,467 alumnos de quinto y sexto grado.

Los municipios de Juárez y Chihuahua tienen la peculiaridad de ser los más den- sos en población del estado de Chihuahua, México, ya que concentran el $63.5 \%$ de sus ciudadanos (INEGI 2010), asunto que fue tomado en cuenta para su selección y de ahí obtener las muestras representativas de docentes, directores, ATP y supervisores o inspectores, de acuerdo a la factibilidad de recursos financieros y humanos de la investigación emprendida.

De esta cantidad de actores consultados, los que contestaron su origen estatal por su función son 525 docentes (el 47.7\%), 54 directores (el 31.6\%), 6 ATP que representa el $5.7 \%$ y 13 inspectores que corresponde al $18.8 \%$; en cambio los federalizados son: 575 docentes (el 51.8\%), 115 directores (el 68\%), 94 ATP que representan el $88.7 \%$ y 53 supervisores escolares equivalente al $76.8 \%$ como se muestra en la Tabla 1.

Tabla 1. Actores consultados distribuidos por función y origen laboral

\begin{tabular}{|l|l|l|l|l|l|l|l|l|l|l|}
\hline & \multicolumn{2}{|c|}{ Docentes } & \multicolumn{2}{c|}{ Directivos } & \multicolumn{2}{c|}{ ATPs } & \multicolumn{2}{c|}{ Supervisores } & \multicolumn{3}{c|}{ Total } \\
\hline & Frec & $\%$ & Frec & $\%$ & Frec & $\%$ & Frec & $\%$ & Frec & $\%$ \\
\hline Estatales & 525 & 47.7 & 54 & 31.6 & 6 & 5.7 & 13 & 18.8 & 598 & 41.1 \\
\hline Federales & 575 & 51.8 & 115 & 68.0 & 94 & 88.7 & 53 & 76.8 & 837 & 57.5 \\
\hline N.C. & 12 & 1.1 & 0 & 0.0 & 6 & 5.7 & 3 & 4.3 & 21 & 1.44 \\
\hline Total & 1112 & 100 & 169 & 99.6 & 106 & 100 & 69 & 99.9 & 1456 & 100 \\
\hline
\end{tabular}

\section{Resultados}

\section{Algunas percepciones sobre su condición laboral}

El 55\% de los docentes frente a grupo declararon estar en desacuerdo o totalmente en desacuerdo ante la afirmación de si "las condiciones laborales han mejorado a partir de la implementación de la RIEB”. El $24.7 \%$ se coloca en posición neutral y sólo el $15.7 \%$ estuvieron de acuerdo o totalmente de acuerdo con la afirmación.

Este ítem, como se muestra en la Gráfica 1 , también fue planteada a los directores y sus respuestas se orientaron en un $61.6 \%$ en desacuerdo y totalmente en desacuerdo, el 22.5\% quedó en posición neutral mientras que el $13.1 \%$, estuvieron de acuerdo o totalmente de acuerdo. 


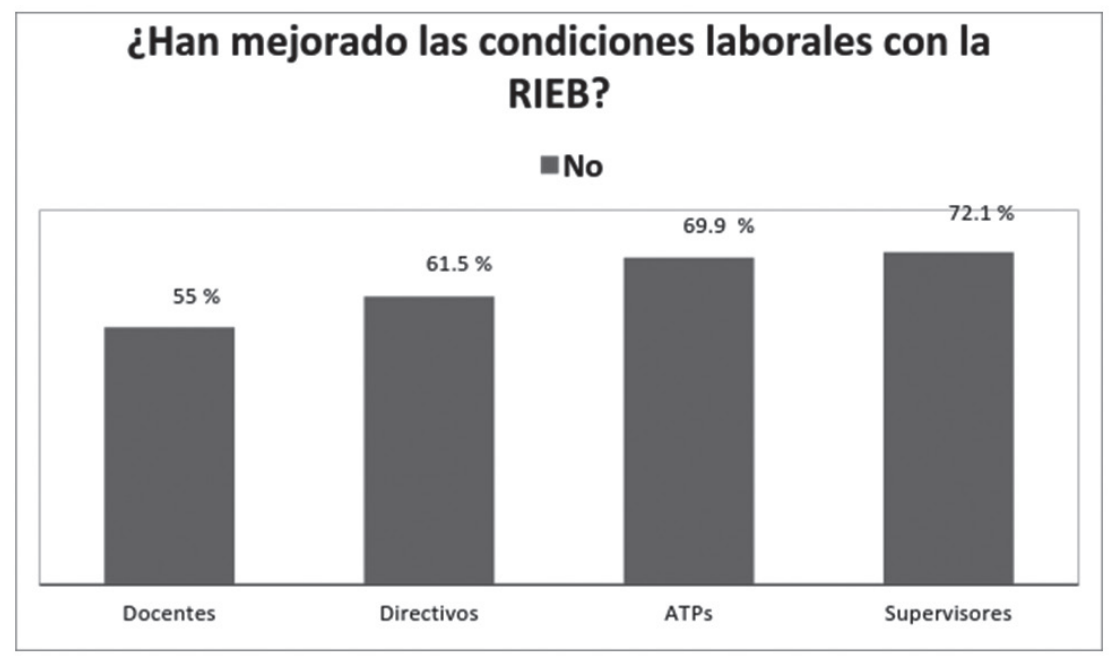

Gráfica 1. No han mejorado las condiciones laborales con la RIEB

Los asesores técnico pedagógicos en un 69.9\% también están en desacuerdo y totalmente en desacuerdo con la afirmación de que las condiciones laborales de los docentes han mejorado a partir de la implementación de la RIEB; a la vez solo 2 casos estuvieron de acuerdo con dicha aseveración.

Y el porcentaje en sentido negativo se incrementa aún más ante la percepción de los supervisión, inspector o jefe de sector ya que el $72.1 \%$ consideraron estar en desacuerdo o totalmente en desacuerdo con la afirmación de que "las condiciones laborales han mejorado a partir de la implementación de la RIEB".

En cierta modo, la información anterior se confirma en las respuestas otorgadas por los consultados a la pregunta: ¿Las exigencias en el trabajo docente son mayores a partir de la implementación de la RIEB?, ya que un $55.6 \%$ de los docentes contestaron estar de acuerdo o totalmente de acuerdo; de igual forma los directores lo indicaron en ese mismo sentido en un 58.5\%; y lo reiteraron de forma más contundente los asesores técnico pedagógicos con un $71.7 \%$, al igual que los supervisores con un $73.9 \%$, tal cual se muestra en la Gráfica 2.

\section{Las exigencias en el trabajo docente son mayores a partir de la RIEB}

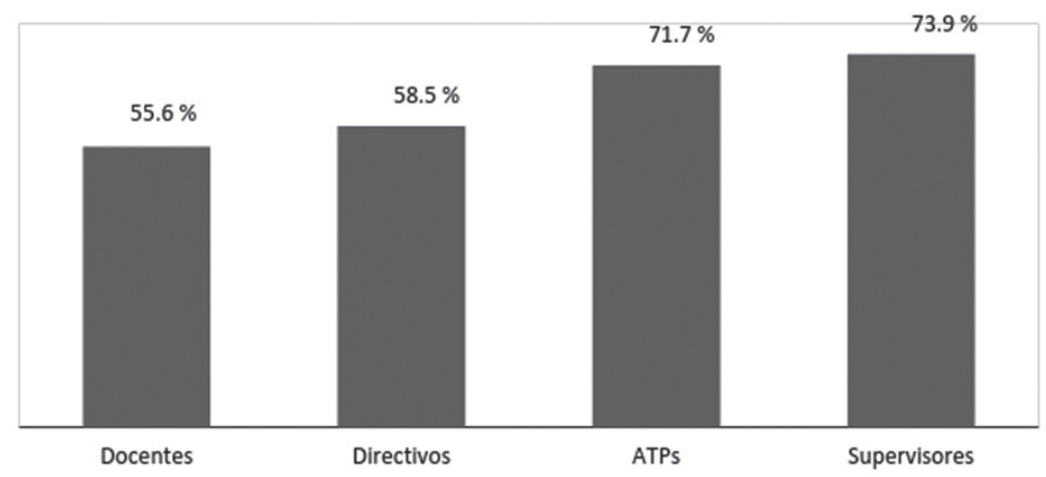

Gráfica 2. Exigencias mayores a los docentes, a partir de la RIEB

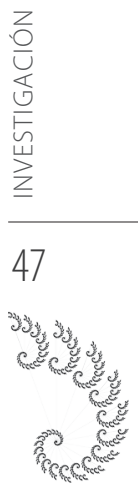




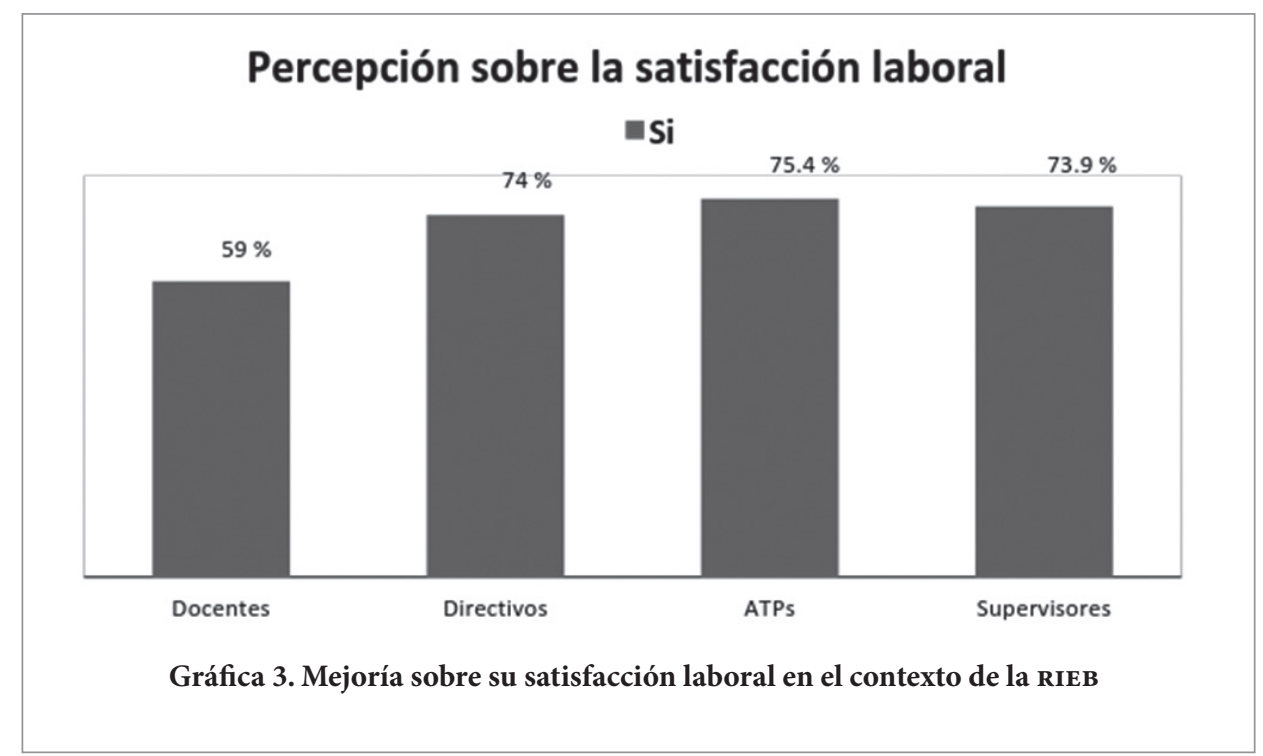

A la percepción en sentido negativo de los actores consultados sobre la mejoría de las condiciones laborales y la percepción de que existen mayores exigencias laborales ante la RIEB, se le adiciona la percepción positiva sobre su satisfacción laboral ilustrada en la Gráfica 3.

Así, sobre la precepción que guardan en relación a la satisfacción laboral en el contexto de la RIEB, el 59\% de los docentes frente a grupo de los dos municipios más poblados del estado de Chihuahua además del de Ahumada, consideró que su satisfacción laboral ha mejorado notablemente o simplemente ha mejorado, el $24.1 \%$ considera que sigue igual y solo el $10.4 \%$ de los casos afirman que ha empeorado de forma notoria o simplemente empeorado.

En el caso de los directores, el 74\% afirman que su satisfacción laboral ha mejorado notablemente o simplemente ha mejorado; además, el 75.4\% de ATP también lo refieren en este sentido, y de los supervisores, inspectores o jefes de sector, el 75\% tienen la misma percepción.
El análisis de las respuestas a estos tres ítems nos refleja que por un lado, la percepción mayoritaria se orienta a que las condiciones laborales no han mejorado en el contexto de la RIEB y que en cambio, las exigencias sobre su labor sí han aumentado, pero su percepción sobre la satisfacción laboral que viven en este contexto ha mejorado notablemente o simplemente ha mejorado; esto puede ser posible por el hecho de que, si no mejoraron las condiciones laborales, no implica que hayan sido malas, es decir, pueden ser buenas de origen y seguir igual, aunque no hayan mejorado más.

Otro ítem que fue aplicado a cada uno de los consultados y que se enfocó hacia su valoración personal sobre la función que cada quien desempeña, el 57.7\% de los docentes frente a grupo afirman que han mejorado notoriamente o simplemente mejorado, es decir, expresan una valoración favorable de su función; así también, el 68.6\% de los directores lo afirman en este mismo sentido en tanto el $71.7 \%$ de los ATP respondieron de forma semejante, y el 76.5\% 


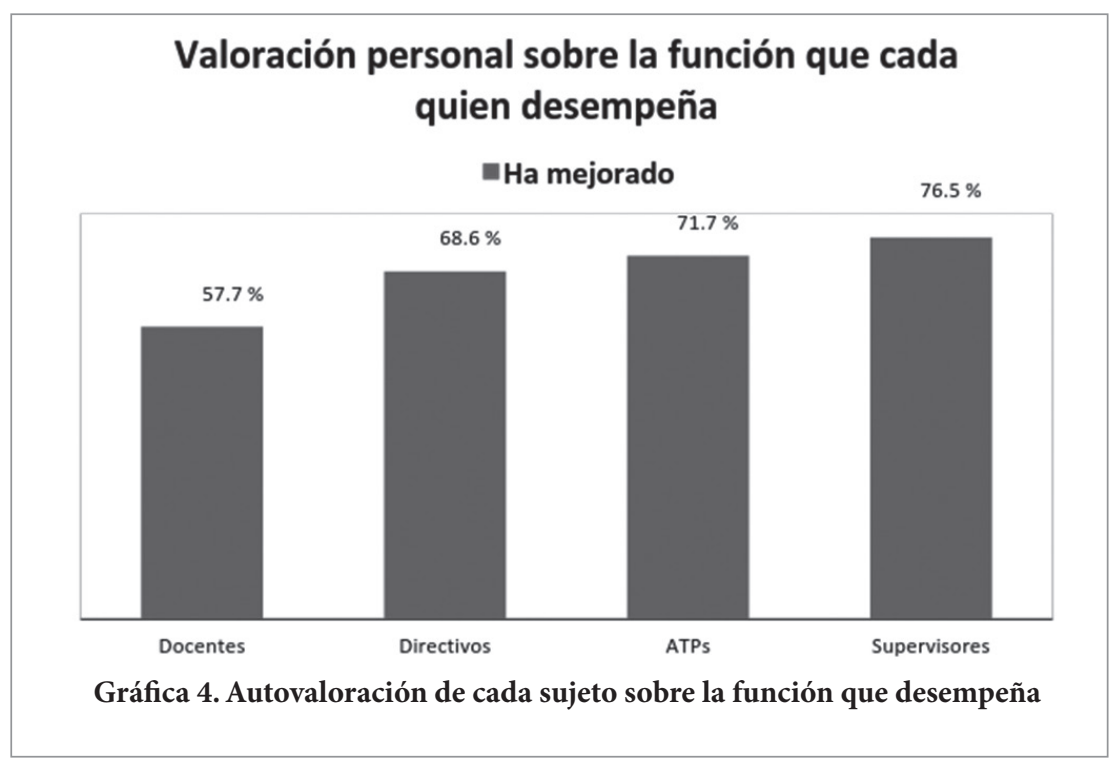

de los supervisores, inspectores o jefes de sector también asumen ésta tendencia.

Como se observa en la Gráfica 4, en las cuatro diferentes funciones las respuestas fueron en ascenso porcentualmente hablando a favor de una valoración personal positiva sobre su función.

Los docentes en un $57.7 \%$, los directores en un $68.6 \%$, los ATP en un $71.7 \%$ y los supervisores en un $76.5 \%$ valoran que la función que desempeñan ha mejorado o mejorado notoriamente en el contexto de la RIEB, resultado que corresponde con la percepción que tienen de su satisfacción laboral que también se presenta aproximadamente con esa tendencia en el mismo orden: $59 \%, 74 \%, 75.4 \%$ y $73.9 \%$

El reconocer en sentido mayoritariamente positivo que las condiciones laborales no han mejorado con la implementación de la RIEB, no impide que ellos hagan una valoración positiva del desempeño de su función, que implica el auto reconocimiento de su esfuerzo con buenos resultados.
En este sentido, el hecho de que no hayan mejorado sus condiciones laborales no implica que hayan empeorado. Tener más trabajo no necesariamente se traduce en insatisfacción puesto que por la naturaleza novedosa del mismo puede significarles un reto, y al enfrentarle positivamente, puede traducirse en mayor satisfacción como se muestra en este análisis.

\section{Conclusiones}

Estos resultados nos indican que los docentes de educación primaria, tanto federalizados como estatales de los municipios de Ahumada, Chihuahua y Juárez, en el contexto de la implementación de la Reforma Integral de la Educación Básica perciben que las exigencias laborales y profesionales realmente han aumentado, y que a pesar de ello, las condiciones laborales que tenían antes de esta reforma siguen sin cambio hacia un mejor estadio.

A la vez, en sus funciones de docente frente a grupo, director/a de escuela, de asesoría técnico pedagógica, de supervisor/a, 
REVISTA DE INVESTIGACIÓN EDUCATIVA DE LA REDIECH N. 8

ISSN: 2007-4336

inspector/a o jefe/a de sector, coinciden en expresar que su percepción sobre su satisfacción laboral es positiva además de que su propia valoración sobre su desempeño laboral también es positiva.

Estos elementos derivados del análisis de los datos empíricos nos presentan algunos efectos positivos de la implementación de la reforma en cuestión en las percepciones de los consultados; se aprecia que los niveles de resistencia a la misma son reducidos y que es una mayoría la que ha asumido la propuesta lo cual a la vez que es un balance positivo propicia condiciones apropiadas para su consecución; esto es un dato importante que arroja la presente investigación; si hay satisfacción laboral positiva en seis o siete de cada diez de los consultados y a la vez hay una valoración positiva de su función que desempeñan esos seis o siete de cada diez, es indicativo de un avance consistente a cuatro años de la implementación de la RIEB, que fue cuando se recogió la información. Habrá que revisar si esto mismo lo perciben los alumnos y los padres de familia, asunto de otra investigación.

\section{Referencias}

Fuentes Reza, R. (2010). Las condiciones laborales y profesionales de los docentes de las escuelas secundarias. Tesis para obtención de grado de Maestría en Educación. Centro de Investigación y Docencia. Chihuahua, México.

INEGI, 2010. Instituto Nacional de Estadística y Geografía. Consultado el 5 de mayo de 2013 en: http:// www.inegi.org.mx

Ley Federal de Trabajadores al Servicio del Estado, Reglamentaria del Apartado B) del Artículo 123 Constitucional (DOF: 02042014).

Oliveira D., Goncalves G., Melo S. (2004). Cambios en la organización del trabajo docente. Consecuencias para los profesores. Revista Mexicana de Investigación Educativa COMIE, 020, 188. Consultado el 22 de octubre de 2010 en www.redalyc.com

Reglamento de las Condiciones Generales de Trabajo del Personal de la Secretaría de Educación Pública (1946).

\section{Agradecimientos}

A las autoridades educativas de los dos subsistemas de educación primaria del Estado de Chihuahua por su respaldo y autorización para el trabajo de campo, a los profesores y profesoras que participaron como informantes por su valiosa colaboración, a los estudiantes de los grupos de maestría del Centro de Investigación y Docencia de Ahumada, Chihuahua y Juárez Generación 18 y 20 en calidad de colaboradores de la investigación y al Cuerpo Académico de Política y Gestión en Educación por su visión estratégica y concreción de su programa de trabajo. 